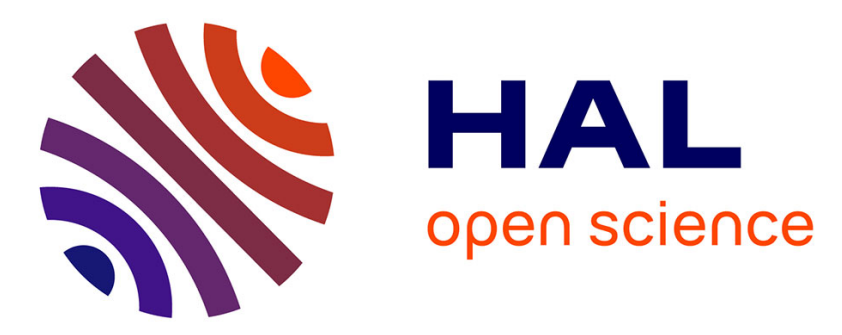

\title{
Control of a teleoperation system actuated by low-cost pneumatic on/off valves
}

\author{
Sean Hodgson, Mahdi Tavakoli, Arnaud Lelevé, Minh Tu Pham
}

\section{To cite this version:}

Sean Hodgson, Mahdi Tavakoli, Arnaud Lelevé, Minh Tu Pham. Control of a teleoperation system actuated by low-cost pneumatic on/off valves. 2012 IEEE IROS, Oct 2012, Vilamoura, Portugal. pp.3029 - 3034, 10.1109/IROS.2012.6385697 . hal-00926988

\section{HAL Id: hal-00926988 \\ https://hal.science/hal-00926988}

Submitted on 29 Jan 2016

HAL is a multi-disciplinary open access archive for the deposit and dissemination of scientific research documents, whether they are published or not. The documents may come from teaching and research institutions in France or abroad, or from public or private research centers.
L'archive ouverte pluridisciplinaire HAL, est destinée au dépôt et à la diffusion de documents scientifiques de niveau recherche, publiés ou non, émanant des établissements d'enseignement et de recherche français ou étrangers, des laboratoires publics ou privés. 


\title{
Control of a teleoperation system actuated by low-cost pneumatic on/off
} valves

\author{
Sean Hodgson ${ }^{1}$, Mahdi Tavakoli ${ }^{1}$, Arnaud Lelevé ${ }^{2}$, Minh Tu Pham ${ }^{2}$ \\ ${ }^{1}$ Department of Electrical and Computer Engineering, University of Alberta, Edmonton, AB Canada T6G2V4 \\ ${ }^{2}$ Laboratoire Ampère, UMR CNRS 5005, Université de Lyon, INSA-LYON, F-69621 Villeurbanne Cedex, France
}

Abstract-For a pneumatic teleoperation system, slidingmode control laws ensuring both transparency and low switching (open/close) activity of the valves are developed. Each pneumatic actuator has four on/off valves, thus sixteen possible combinations ("operating modes") for the valves exist but only seven of them are both functional and unique. While previous works have focused on three-mode sliding-based position control of one pneumatic actuator, this paper develops seven-mode sliding-based bilateral control of a teleoperation system comprising a pair of pneumatic actuators. The proposed bilateral sliding control scheme is experimentally validated on a pair of actuators arranged in a force-position teleoperation architecture. The results demonstrate that leveraging the additional modes of operation leads to more efficient and smooth control of the system.

Index Terms-Teleoperation system, transparency, slidingmode control, pneumatic actuator.

\section{INTRODUCTION}

Teleoperation aims to allow a human operator to carry out a sensing or manipulation task in an environment that is not amenable to direct interaction. The interaction between the human, the teleoperator, and the environment needs to be controlled in such a way as to ensure a high level of "fidelity," which is defined as accurate transmission of the environment's mechanical properties to the human operator [1]. Bilateral teleoperation systems have been developed for a variety of applications ranging from telesurgery to space exploration [2], [3], [4].

In this paper, we investigate the usefulness of low-cost pneumatic components when used as actuators in teleoperation systems. Pneumatic actuators offer many advantages for positioning applications such as low maintenance cost, high ratio of power to weight, cleanliness, and safety [5]. Also, where it is not possible to use electric motors such as in robot-assisted surgery under MRI guidance, it is possible to use pneumatic actuators to drive robots. On the down side, pneumatic actuators typically suffer from stiction and sensitivity of the actuator dynamics to external loading and piston position along the cylinder stroke [6]. Thus, designing an effective control architecture for a pneumatic actuator is fairly challenging and this is exacerbated by the nonlinear pressure dynamics in pneumatic chambers.

Some pneumatic systems utilize servo-valves, which allow for a continuous change of the input mass flow rate [7]. However, servo-valves are expensive devices. A low-cost substitute to the servo-valve is the on/off (i.e., open/close) solenoid valve, which does not require the expensive components used in manufacturing a servo-valve. However, due to the discrete-input nature of the on/off solenoid valve, it cannot use continuous control laws as was the case with the servo-valve. The input discontinuity makes the nonlinear dynamics of the pneumatic actuator harder to control.

Previous research has investigated linearizing the nonlinear dynamics of a solenoid-valve pneumatic actuator to generate an equivalent linear system [5]. Past research has also studied using solenoid-valve pneumatic actuators with a pulse width modulated (PWM) input [8]. A PWM input with a sufficiently high frequency to a solenoid valve is deemed to approximate the continuous input properties of a servo-valve [9].

When considering a single pneumatic actuator that is comprised of two chambers and four on/off solenoid valves, one would assume that there would be a total of sixteen distinct combinations of valves' on or off positions ("operating modes"). Each chamber has two solenoid valves, one that can connect to a supply pressure, and one that can connect to an exhaust pressure. We do not want to connect a chamber to both pressure and exhaust at the same time, therefore we find that seven of the sixteen possible valve combinations are invalid. Of the remaining nine modes, three can be considered functionally equivalent under no-load conditions. Thus, the system has a total of seven unique discrete modes.

Sliding-mode control is a nonlinear control method that can be utilized for discrete-input (and continuous-input) systems. It alters the dynamics of the system by applying a high-frequency switching control signal [10]. A major strength of this method lies in its parametric robustness; since it performs a simple switching between two operating modes, the control law will not be sensitive to the open-loop system's parameter variations or nonlinearities [11].

In [11], a sliding-mode control based on three of the aforementioned discrete modes was applied to a two-chamber solenoid-valve actuator. Good tracking and relatively low steady-state position errors were shown. In [12], it has been demonstrated that for a single pneumatic actuator, expanding the control possibilities from three-mode control to seven-mode control reduces the tracking error and the valves' switching activity, causing an overall improvement in the pneumatic system performance. In this paper, we design a seven-mode based sliding control for force-position control of a bilateral teleoperation system. It is expected that 
the four additional modes of operation help to utilize just the necessary amounts of drive energy, allowing smoother teleoperation control. To assess this conjecture, we compare teleoperation performance under 3-mode sliding control versus 7-mode control.

The organization of this paper is as follows. The discreteinput mode-based model of a single pneumatic actuator with solenoid valves is given in Section II. Sliding-mode control of the single pneumatic actuator is discussed in Section III. Sliding-mode control of a teleoperated pair of pneumatic actuators is reported in Section IV. The experimental results are reported in Section V. Finally, the concluding remarks and directions for future research are presented in Section VI.

\section{Discrete-InPut Model of The Open-Loop} ACTUATOR

We consider a pneumatic actuator comprised of two chambers as shown in Figure 1. Each chamber is connected via its two solenoid valves to either the supply pressure or the exhaust pressure. In the Appendix, the model of the open-loop pneumatic actuator has been derived. In general, the usage of more modes by the controller is expected to facilitate the use of more optimal amounts of drive energy, meaning that improved positioning precision and reduced valve switching (open/close) activity should result. This paper studies this issue in the context of teleoperation control of a pair of pneumatic actuators equipped with solenoid valves. In [11], the following three out of nine discrete modes have been considered for control of the two-chamber solenoid-valve actuator: both chambers locked, one chamber pressurizing and the other chamber venting (and vice versa).

In [13], the three-mode model was expanded to a fivemode model. The five-mode model has two extra modes that utilize the option to have one chamber closed and the other chamber pressurized (and vice versa). In [12], we further extended the five-mode model to a new seven-mode model utilizing the option to have one chamber closed and the other chamber vented (and vice versa). With each extension of the controlled modes, the performance was found to improve while controller complexity increased.

We mentioned previously that there are a total of nine discrete modes for the solenoid valves. The question is whether there is any significant advantage in further expansion of control possibilities from seven to the full nine modes. The nine possible modes are shown in Table I. The discrete voltage inputs $U_{1}, U_{2}, U_{3}$, and $U_{4}$ open or close the actuator's solenoid valves and are shown in Figure 1. Note that $U_{i} \in\{0,1\}$, where 0 refers to a closed valve and 1 refers to an open valve. Reviewing these different discrete modes, we observe that the modes $M_{1}, M_{8}$, and $M_{9}$ are functionally similar (under no load) as they correspond to the two chambers being both closed, both venting, and both pressurized, respectively. For all of these three modes, the pressure difference across the chamber $\mathrm{P}$ and chamber $\mathrm{N}$ is reduced to zero over time, implying that the piston acceleration profile will be similar for them. In our research, the mode $M_{1}$ was selected out of the three equivalent modes $\left(M_{1}, M_{8}\right.$, and $\left.M_{9}\right)$ because it provides the highest resistance to piston motion, which will facilitate reducing the position tracking error to zero. Thus, our modeling and control analysis from this point forward will focus on the modes $M_{1}$ to $M_{7}$.

\section{TABLE I}

Nine Discrete Modes of The Open-Loop Actuator

\begin{tabular}{cccccccc|cc}
\hline \hline & $\mathrm{M}_{1}$ & $\mathrm{M}_{2}$ & $\mathrm{M}_{3}$ & $\mathrm{M}_{4}$ & $\mathrm{M}_{5}$ & $\mathrm{M}_{6}$ & $\mathrm{M}_{7}$ & $\mathrm{M}_{8}$ & $\mathrm{M}_{9}$ \\
\hline $\mathrm{U}_{1}$ & 0 & 1 & 0 & 0 & 0 & 1 & 0 & 0 & 1 \\
\hline $\mathrm{U}_{2}$ & 0 & 0 & 1 & 0 & 0 & 0 & 1 & 1 & 0 \\
\hline $\mathrm{U}_{3}$ & 0 & 0 & 0 & 0 & 1 & 0 & 1 & 0 & 1 \\
\hline $\mathrm{U}_{4}$ & 0 & 0 & 0 & 1 & 0 & 1 & 0 & 1 & 0 \\
\hline \hline
\end{tabular}

As detailed in the Appendix, the dynamics of the pneumatic actuator of Figure 1 involving the chamber pressures and the resulting piston motion is

$$
\left(A_{P} P_{P}-A_{N} P_{N}\right)-b \dot{y}-\tau_{S t}+\tau_{E x t}=M \ddot{y}
$$

where $P_{P}$ and $P_{N}$ refer to pressures $(P a)$ inside the chambers $P$ and $N$, respectively; $A_{P}$ and $A_{N}$ refer to the piston cylinder areas $\left(\mathrm{m}^{2}\right)$ in the chambers $\mathrm{P}$ and $\mathrm{N}$, respectively; $\mathrm{b}$ is the viscosity coefficient $(\mathrm{N} \mathrm{s} / \mathrm{m}), M$ is the total mass of the load and the piston $(\mathrm{Kg}), \tau_{S t}$ is the stiction force $(\mathrm{N}), \tau_{E \times t}$ is the external force $(\mathrm{N})$, and $\mathrm{y}$ refers to the piston position $(\mathrm{m})$ shown in Figure 1. Note the arrows for position $y$ and force $F_{E x t}$ and $F_{S t}$ in Figure 1 refer to their positive directions. The stiction force $\tau_{S t}$ was considered to be negligible since the pneumatic actuator used in our experiments was an Airpel anti-stiction cylinder (All Air Inc., New York, USA).

It is possible to find the switched dynamical equation for the 7-mode system by taking the time derivative of (1) and substituting the pressure dynamics $\dot{P}_{\mathrm{P}}$ and $\dot{P}_{N}$ (see Appendix).

$$
\dddot{y}=\begin{array}{ll}
f+\frac{\dot{t}_{E \times t}}{M} & , \text { mode } M_{1} \\
f+(-1)^{j} g+\frac{\dot{t}_{E \times t}}{M} & , \text { mode } M_{j}=M_{1}
\end{array}
$$

where the integer $\mathrm{j}$ ranges from 2 to 7 and

$$
\begin{array}{cc}
f=\frac{-b}{M} \ddot{y}-\frac{k}{M} & \frac{A_{P} P_{P}}{l / 2+y}+\frac{A_{N} P_{N}}{l / 2-y} \quad \dot{y} \\
g_{2}=\frac{k r T}{M} \frac{Q\left(P_{S}, P_{P}\right)}{(I / 2+y)} & g_{3}=\frac{k r T}{M} \frac{Q\left(P_{P}, P_{E}\right)}{(I / 2+y)} \\
g_{4}=\frac{k r T}{M} \frac{Q\left(P_{N}, P_{E}\right)}{(I / 2-y)} & g_{5}=\frac{k r T}{M} \frac{Q\left(P_{S}, P_{N}\right)}{(I / 2-y)} \\
g_{6}=g_{2}+g_{4} & g_{7}=g_{5}+g_{3}
\end{array}
$$

In the above, $P_{S}$ and $P_{E}$ are the pressures of the compressed air supply and the exhaust (atmosphere), respectively; $I$ is the total length of the chamber, $\mathrm{k}$ refers to the polytropic constant, $\mathrm{T}$ is the supply temperature, and $\mathrm{r}$ refers to the universal gas constant $(J /(\mathrm{kg} . \mathrm{K}))$. In general, $Q\left(P_{U p}, P_{D \text { own }}\right)$, in which $P_{U p}$ is the upstream pressure and $P_{D \text { own }}$ is the downstream pressure, refers to the expression for the mass 


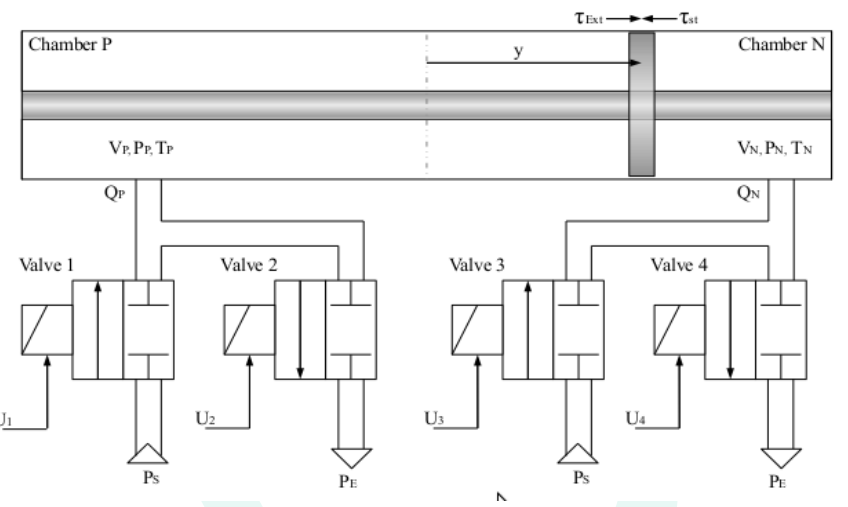

Fig. 1. Single pneumatic actuator with 4 on/off solenoid valves

flow rate through an orifice.

It should be noted that the functions $g_{2}$ through $g_{7}$ are all positive or equal to zero.

\section{Sliding-mode Control of a Pneumatic ACTUATOR}

For a position controlled system, let us define a sliding surface $S=0$ where the switching function $S$ is defined as

$$
\mathrm{s}=\frac{\ddot{\mathrm{e}}}{\omega^{2}}+\frac{2 \xi \dot{\mathrm{e}}}{\omega}+\mathrm{e}
$$

Here, $\mathrm{y}$ is the piston position, $\mathrm{y}_{\mathrm{d}}$ is the piston desired position, $e=y-y_{d}$ is the piston position error, and $\xi$ and $\omega$ are constant and positive numbers. The switching function $\mathrm{S}$ provides a measure of the distance from the sliding surface based on the position, velocity, acceleration errors. Take the derivative of (3) to get

$$
\begin{aligned}
\dot{s} & =\frac{\dddot{\mathrm{e}}}{\omega^{2}}+\frac{2 \xi \ddot{\mathrm{e}}}{\omega}+\dot{\mathrm{e}} \\
& =\frac{\dddot{\mathrm{y}}-\dddot{y}_{\mathrm{d}}}{\omega^{2}}+\frac{2 \xi \ddot{\mathrm{e}}}{\omega}+\dot{\mathrm{e}}
\end{aligned}
$$

Now, for our pneumatic actuator, by substituting the actuator dynamics (2) into (4) we obtain

$$
\dot{s}=\begin{array}{ll}
\lambda & , \text { mode } M_{1} \\
\lambda+(-1)^{i} g_{i} / \omega^{2} & , \text { mode } M_{i},(2 \leq i \leq 7)
\end{array}
$$

where $\lambda=\left(f-\dddot{y}_{d}+\dot{\tau}_{E x t} / M\right) / \omega^{2}+2 \xi \ddot{e} / \omega+\dot{e}$

To ensure the convergence to the sliding surface $S=0$, we wish to have $\dot{S}$ such that $\mathbf{S}$ is always approaching zero regardless of its sign. To this end, we will invoke the seven different modes of the open-loop system based on the current value of $S$ relative to five distinct regions (ranges) for the value of $\mathrm{S}$. These regions of $\mathrm{S}$ and the operating mode of the system as selected by the sliding-mode controller are illustrated in Table II. This ensures $\dot{S}<0$ when $s>0$ and $\dot{s}>0$ when $s<0$, leading to the convergence to $s=0$.

When we utilize the pneumatic controller that alters the operating mode of the pneumatic actuator system based on Table II, for the lowest error band $|\mathrm{s}|<$, we use the mode $M_{1}$ which has no active effect on the system (i.e., no actuation). For the highest positive error band $s>\beta$, we
SELECTION OF THE OPERATING MODE BASED ON THE VALUE OF $\mathbf{S}$.

\begin{tabular}{ccc}
\hline \hline Region of $S$ & $\begin{array}{c}\text { Selected } \\
\text { operating mode }\end{array}$ & $\begin{array}{c}\text { Magnitude of resulting } \\
\text { s from (5) }\end{array}$ \\
\hline$S>\beta$ & $M_{7}$ & Large negative \\
$\beta \geq S>M$ & 3 and $M_{5}$ & Medium negative \\
$\geq S>-M$ & 1 & Small \\
$-\geq S>-\beta$ & $M_{2}$ and $M_{4}$ & Medium positive \\
$-\beta \geq S$ & $M_{6}$ & Large positive \\
\hline \hline
\end{tabular}

TABLE III

MASTER/SLAVE ACTUATOR VARIABLE NAMES WHERE $q \in\{P, N\}$, $v \in\{1, \ldots, 7\}$, AND $t \in\{1, \ldots, 7\}$.

\begin{tabular}{|c|c|c|c|c|c|}
\hline Single & $\mathrm{y}$ & $\mathrm{P}_{\mathrm{q}}$ & $\tau_{\mathrm{E} x \mathrm{t}}$ & $\mathrm{g}_{\mathrm{i}}$ & $\mathrm{f}$ \\
\hline Master & $\mathrm{y}_{\mathrm{m}}$ & $\mathrm{P}_{\mathrm{q}, \mathrm{m}}$ & $\tau_{\mathrm{h}}$ & $\mathrm{g}_{\mathrm{v}, \mathrm{m}}$ & $\mathrm{f}_{\mathrm{m}}$ \\
\hline Slave & $\mathrm{y}_{\mathrm{s}}$ & $\mathrm{P}_{\mathrm{q}, \mathrm{s}}$ & $-\tau_{\mathrm{e}}$ & $\mathrm{g}_{\mathrm{t}, \mathrm{s}}$ & $\mathrm{f}_{\mathrm{s}}$ \\
\hline
\end{tabular}

use the mode $M_{7}$, which exerts the highest drive (causing the highest piston acceleration) in the negative direction. Conversely, for the largest negative error $s \leq-\beta$, we utilize mode $M_{6}$, which has the highest drive in the positive direction [12]. Evidently, this control action is designed to ensure that the system is always approaching the $\mathrm{S}=0$ surface.

In the next section, we will be applying the seven-mode control to a teleoperated pair of actuators under forceposition control.

\section{Sliding-Mode Control of a Dual Pneumatic TELEOPERATION SYSTEM}

For a teleoperation system, the master and slave dynamics will be the same as those described previously with the difference that the common variables will be re-labeled as shown in Table III.

The block diagram in Figure 2 shows the architecture of a force-position bilateral teleoperation system. In this setup, the human operator dynamics, $Z_{\mathrm{h}}$, and the environment dynamics, $Z_{e}$, are unknown or uncertain; and $\tau_{h}$ and $\tau_{e}$ are the operator force applied on the master and the environmental force applied on the slave, respectively. Also, $\tau_{h}^{*}$ and $\tau_{e}^{*}$ are continuous exogenous input forces from the operator and the environment, which have limited energy and as such are bounded.

The dynamics for the master and the slave are

$$
\begin{array}{r}
M \ddot{y}_{m}=A\left(P_{P, m}-P_{N, m}\right)-b \dot{y}_{m}+\tau_{h} \\
M \ddot{y}_{s}=A\left(P_{P, s}-P_{N, s}\right)-b v \dot{y}_{s}-\tau_{e}
\end{array}
$$

Substituting the variables from Table III into (2) we obtain

$$
\begin{aligned}
& \dddot{y}_{m}=\begin{array}{ll}
f_{m}+\dot{\tau}_{h} / M & , \operatorname{mode} M_{1} \\
f_{m}+(-1)^{v} g_{v, m}+\dot{\tau}_{h} / M & , \text { mode } M_{v}=M_{1}
\end{array} \text { mode } M_{1} \\
& \dddot{y}_{s}=\begin{array}{ll}
f_{s}-\dot{\tau}_{e} / M & \text { mode } M_{t}=M_{1} \\
f_{s}+(-1)^{t} g_{t, s}-\dot{\tau}_{e} / M
\end{array}
\end{aligned}
$$




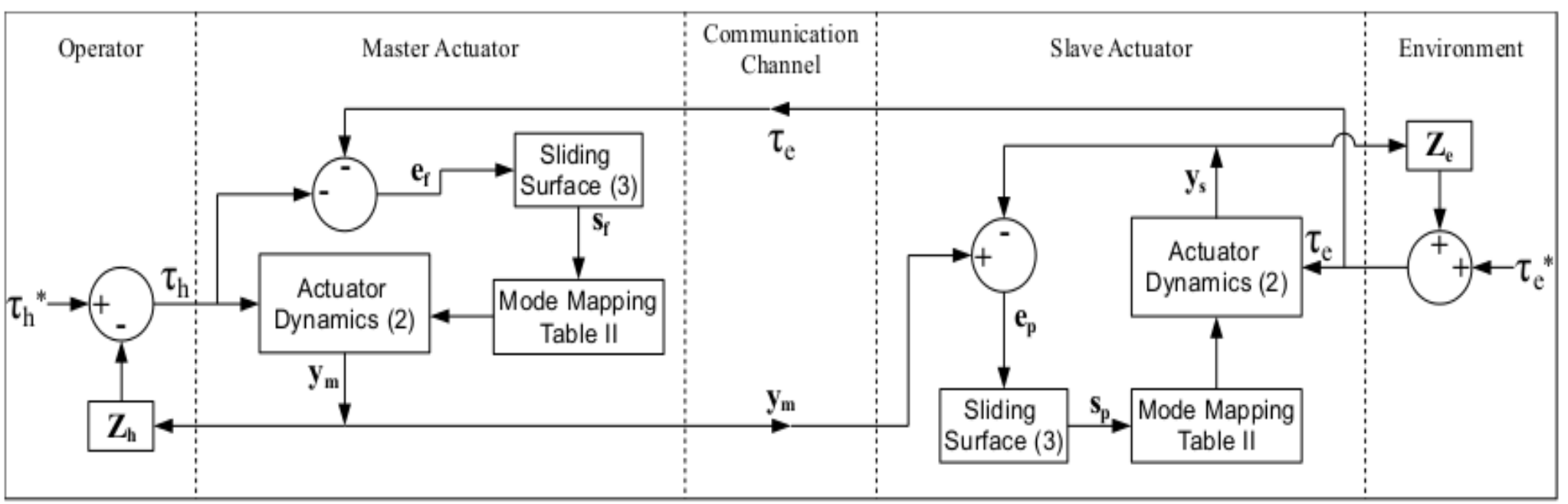

Fig. 2. Force-position based teleoperation.

where $v \in\{1, \ldots, 7\}$ and $t \in\{1, \ldots, 7\}$.

For sliding-mode position (8) and force (9) control purposes, let us define two switching function as

$$
\begin{aligned}
& s_{p}=\frac{\ddot{e}_{p}}{\omega_{p}^{2}}+\frac{2 \xi_{p} \dot{e}_{p}}{\omega_{p}}+e_{p} \\
& s_{f}=\frac{\ddot{e}_{f}}{\omega_{f}^{2}}+\frac{2 \xi_{f} \dot{e}_{f}}{\omega_{f}}+e_{f}
\end{aligned}
$$

in which $e_{p}=y_{m}-y_{s}$ and $e_{f}=-\tau_{h}-\tau_{e}$. It should be noted that the slave controller uses the position-based switching function $\left(S_{p}\right)$ whereas the master controller uses the forcebased switching function $\left(\mathrm{S}_{f}\right)$.

\section{A. Position Tracking Analysis}

To be able to analyze the closed-loop position tracking performance, consider the Lyapunov function candidate

$$
V_{\text {lya }}=\frac{1}{2} s_{p}^{2}
$$

which is positive-definite. Therefore, if $\dot{V}_{\text {lya }}<0$, then $V_{\text {lya }}$ will be decreasing. If $V_{\text {lya }}$ is decreasing, $\left|\mathrm{s}_{\mathrm{p}}\right|$ will also be decreasing. Assuming $S_{p}$ is initially bounded and $\left|S_{p}\right|$ is always decreasing, then $S_{p}$ will always be bounded. This means that $S_{p}$ will approach zero if we control the system so that

$$
\begin{array}{ll}
\dot{s}_{p}>\eta_{p} & \text { if } s_{p}<0 \\
\dot{s}_{p}<-\eta_{p} & \text { if } s_{p}>0
\end{array}
$$

for some positive constant $\eta_{p}>0$ [10].

In the above, $\dot{s}_{p}$ is found by taking the derivative of (8):

$$
\dot{s}_{p}=\frac{\dddot{e}_{p}}{\omega_{p}^{2}}+\frac{2 \xi_{p} \ddot{e}_{p}}{\omega_{p}}+\dot{e}_{p}
$$

Let us consider two possible cases for the sign of $\dot{S_{p}}$ in the following.

- Case 1: Assume that $S_{p}$ is positive. Then, (11) reduces to

$$
\dot{s}<-\eta_{p}
$$

Based on the sliding-mode control outlined in Section III (specifically, see Table II), the master and slave systems' closed-loop dynamics become

$$
\begin{gathered}
\dddot{y}_{m}=f_{m}+(-1)^{v} g_{v, m}+\dot{\tau}_{h} / M \quad v \in\{1-7\} \\
\dddot{y}_{s}=f_{s}+g_{t, s}-\dot{\tau}_{e} / M \quad t \in\{2,4,6\}
\end{gathered}
$$

Given

$$
\begin{aligned}
\dddot{e}_{p} & =\dddot{y}_{m}-\dddot{y}_{s} \\
& =\left(f_{m}-f_{s}\right)-\left(g_{t, s}-(-1)^{v} g_{v, m}\right)+\left(\dot{\tau}_{h}+\dot{\tau}_{e}\right) / M
\end{aligned}
$$

Substituting (15) into (12), we find

$$
\dot{s}_{p}=\lambda_{p}-g_{t, s} / \omega_{p}^{2}
$$

where

$\lambda_{p}=\frac{\left(f_{m}-f_{s}\right)+(-1)^{v} g_{v, m}+\left(\dot{\tau}_{h}+\dot{\tau}_{e}\right) / M}{\omega_{p}^{2}}+\frac{2 \xi_{p}}{\omega_{p}} \ddot{e}_{p}+\dot{e}_{p}$

Finally, by substituting (16) into (13) we find that condition (13) is fulfilled if and only if the following condition is met:

$$
g_{t, s}>\left(\lambda_{p}+\eta_{p}\right) \omega_{p}^{2}
$$

- Case 2: Assume that $S_{p}$ is negative. Then, (11) reduces to

$$
\dot{s}>\eta_{p}
$$

Based on the sliding-mode control outlined in Section III (specifically, see Table II), the master and slave systems' closed-loop dynamics become

$$
\begin{gathered}
\dddot{y}_{m}=f_{m}+(-1)^{v} g_{v, m}+\dot{\tau}_{h} / M \quad v \in\{1-7\} \\
\ddot{y}_{s}=f_{s}-g_{t, s}-\dot{\tau}_{e} / M \quad t \in\{3,5,7\}
\end{gathered}
$$

Given 


$$
\begin{aligned}
\dddot{e}_{p} & =\dddot{y}_{m}-\ddot{y}_{s} \\
& =\left(f_{m}-f_{s}\right)+\left(g_{t, s}+(-1)^{v} g_{v, m}\right)+\left(\dot{\tau}_{h}+\dot{\tau}_{e}\right) / M
\end{aligned}
$$

Substituting (21) into (12), we find

$$
\dot{s}_{p}=\lambda_{p}+g_{t, s} / \omega_{p}^{2}
$$

Where $\lambda_{p}$ has been defined in (17).

Also, substituting (22) into (19), we find that condition (19) is fulfilled if and only if the following condition is met:

$$
g_{t, s}>\left(\eta_{p}-\lambda_{p}\right) \omega_{p}^{2}
$$

\section{B. Force Tracking Analysis}

To be able to analyze the force tracking performance, consider the following Lyapunov function candidate

$$
V_{\text {lyb }}=\frac{1}{2} S_{f}^{2}
$$

Evidently, $\mathrm{V}_{\text {lyb }}$ is a positive-definite function and, if $\dot{V}_{\text {lyb }}<$ $0, V_{l y b}$ will be decreasing. If $V_{l y b}$ is decreasing, $\left|\mathrm{s}_{\mathrm{f}}\right|$ will also be decreasing. Assuming $\mathrm{S}_{\mathrm{f}}$ is initially bounded and $\left|\mathrm{S}_{\mathrm{f}}\right|$ is decreasing, $\mathrm{S}_{\mathrm{f}}$ will approach zero. This requires that we control the master robot so that

$$
\begin{array}{ll}
\dot{s}_{f}>\eta_{f} & \text { if } s_{f}<0 \\
\dot{s}_{f}<-\eta_{f} & \text { if } s_{f}>0
\end{array}
$$

for some positive constant $\eta_{\mathrm{f}}>0$.

Take the derivative of (9) to obtain

$$
\dot{s}_{f}=\frac{\dddot{e}_{f}}{\omega_{f}^{2}}+\frac{2 \xi_{f} \ddot{e}_{f}}{\omega_{f}}+\dot{e}_{f}
$$

Let us consider two possible cases for the sign of $\dot{S}_{f}$ in the following.

- Case 1: If we consider that $S_{f}$ is positive, according to (25) we have the following condition

$$
\dot{s}_{\mathrm{f}}<-\eta_{\mathrm{f}}
$$

Based on the sliding-mode control outlined in Section III, the master and slave dynamics become

$$
\begin{array}{cc}
\dddot{y}_{m}=f_{m}-g_{v, m}+\dot{\tau}_{h} / M & v \in\{3,5,7\} \\
\dddot{y}_{s}=f_{s}+(-1)^{t} g_{t, s}-\dot{\tau}_{e} / M & t \in\{1-7\}
\end{array}
$$

Substituting (28) into (26), we find

$$
\dot{s_{f}}=\lambda_{f}-g_{v, m} M
$$

where

$\lambda_{f}=\left(-\dddot{y}_{m}+f_{m}-(-1)^{t} g_{t, s}+\dddot{y}_{s}-f_{s}\right) M+\frac{\dddot{e}_{f}}{\omega_{f}^{2}}+\frac{2 \xi_{f} \ddot{e}_{f}}{\omega_{f}}$

$$
g_{v, m}>\frac{\lambda_{f}+\eta_{f}}{M}
$$

Therefore, (27) is true if and only if condition (31) is met.

- Case 2: If we consider that $S_{f}$ is negative, according to (25) we have the following condition

$$
\dot{s}_{f}>\eta_{f}
$$

Based on the sliding mode control outlined in Section III, the master and slave dynamics become

$$
\begin{array}{cc}
\dddot{y}_{m}=f_{m}+g_{v, m}+\dot{\tau}_{h} / M & v \in\{2,4,6\} \\
\dddot{y}_{s}=f_{s}+(-1)^{t} g_{t, s}-\dot{\tau}_{e} / M & t \in\{1-7\}
\end{array}
$$

Substituting (33) into (26) we find

$$
\dot{s}_{f}=\lambda_{f}+g_{V, m} M
$$

Finally, substituting (34) into (32) we find

$$
g_{v, m}>\frac{\eta_{f}-\lambda_{f}}{M}
$$

Therefore, (32) is true if and only if condition (35) is met.

Therefore, if the positive-valued functions $\mathrm{g}_{\mathrm{i}, \mathrm{m}}$ and $\mathrm{g}_{\mathrm{i}, \mathrm{s}}$ for $i \in\{2-7\}$ are sufficiently large, then the control strategy can satisfy condition (11) (or equivalently (18) and (23)) and condition (25) (or equivalently (31) and (35)) as long as $\lambda_{p}$ and $\lambda_{f}$ are bounded. On the other hand, since all twelve functions $g_{i, m}$ and $g_{i, s}$ are proportional to the valve's mass flow rate, then choosing a large enough valve will ensure that these scalar functions will be sufficiently large and thus the convergence to the sliding surfaces $S_{p}=0$ and $S_{f}=0$ over time is guaranteed.

\section{EXPERIMENTAL RESULTS}

The purpose of this section is to test the advantage offered by 7-mode over 3-mode control in the context of teleoperation control of a pneumatic actuator. We consider the force-position architecture where the slave robot utilizes position control and the master robot utilizes force control. We will compare 3-mode versus 7-mode in terms of position tracking, force tracking, and valve switching activity.

To test the teleoperation control schemes discussed previously, a quasi-periodic input motion pattern was applied by the operator's hand to the master. This input resembled three cycles of back-and-forth motion with an approximately $10 \mathrm{~mm}$ RMS amplitude when the slave was in free space, followed by approximately two seconds of motion causing contact between the slave and its environment, which was a soft material located $14.5 \mathrm{~mm}$ away from the slave's zero position. This entire motion pattern was repeated by the human operator three times over a 20 second period. The position and force profiles of the master and the slave robots were measured via position and force sensors. 


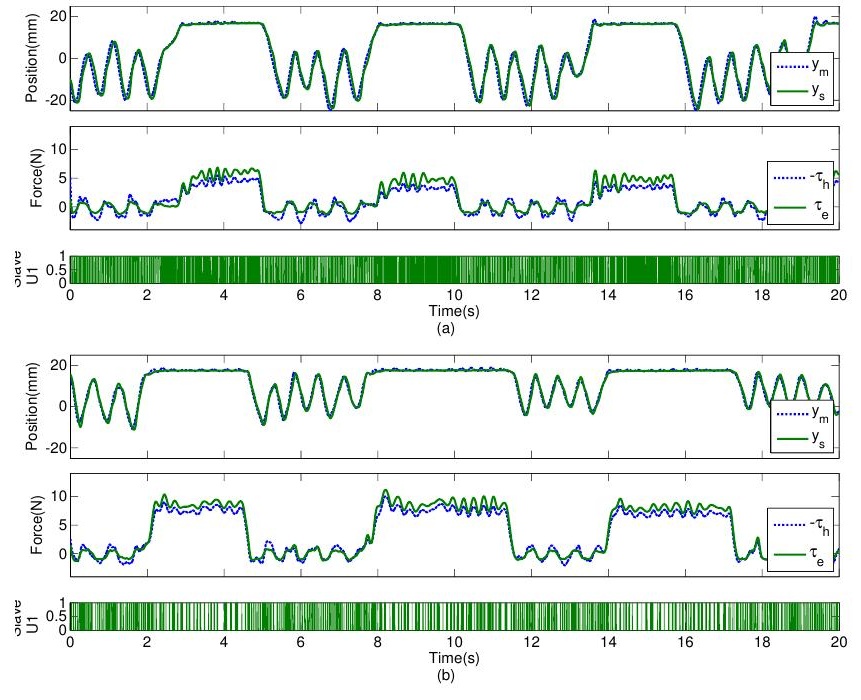

Fig. 3. Experimental setup.

\section{A. Experimental Setup}

In this paper, experiments were performed with a pair of 1-DOF pneumatic actuators as the master and the slave see Figure V-A. The low friction cylinders (Airpel model M16D100D) have a $16 \mathrm{~mm}$ diameter and a $100 \mathrm{~mm}$ stroke. The piston and shaft mass for each actuator is approximately $M=900 \mathrm{~g}$. In terms of sensors, a low-friction linear variable differential transformer (LVDT) is connected to the cylinder in order to measure the linear positions.

The pneumatic solenoid valves (Matrix model GNK821213C3K) used to control the air flow have switching times of approximately $1.3 \mathrm{~ms}$ (opening time) and $0.2 \mathrm{~ms}$ (closing time). With such fast switching times, the on/off valves are appropriate for the purposes of the proposed control.

The controller is implemented using a dSPACE board (DS1104), running at a sampling rate of $500 \mathrm{~Hz}$. This sampling rate has been chosen according to the open/close bandwidth of the valves and to enable an acceptable tracking response. The experimental setup has the model parameters listed in Table IV.

TABLE IV

SYSTEM'S MODEL PARAMETERS.

\begin{tabular}{|c|c|c|}
\hline Var. & Value & Label \\
\hline $\mathrm{I}$ & $0.1 \mathrm{~m}$ & Chamber Length \\
\hline $\mathrm{T}$ & $296 \mathrm{~K}$ & Supply Temperature \\
\hline $\mathrm{C}_{\text {val }}$ & $3.4 \times 10^{-9} \mathrm{~kg} /(\mathrm{s} \mathrm{Pa})$ & Mass Flow Rate Const. \\
\hline $\mathrm{r}$ & $287.0 \mathrm{~J} /(\mathrm{kg} \mathrm{K})$ & Gas Constant \\
\hline $\mathrm{P}_{\mathrm{S}}$ & $300,000 \mathrm{~Pa}$ & Supply Air Pressure \\
\hline $\mathrm{P}_{\mathrm{E}}$ & $100,000 \mathrm{~Pa}$ & Exhaust Air Pressure \\
\hline $\mathrm{k}$ & 1.2 & Polytropic Constant \\
\hline $\mathrm{A}_{\mathrm{P}}, \mathrm{A}_{\mathrm{N}}$ & $1.814 \mathrm{~cm}^{2}$ & Piston Cylinder Area \\
\hline $\mathrm{b}$ & $50 \mathrm{~N} \mathrm{~s} / \mathrm{m}$ & Viscosity Coefficient \\
\hline $\mathrm{M}$ & $0.9 \mathrm{~kg}$ & Total Mass of load \\
\hline
\end{tabular}

Fig. 4. Position and force tracking profiles for the master and the slave robots in force-position architecture: (a) with 3-Mode sliding control and (b) with 7-Mode sliding control.

\section{B. Force-Position Teleoperation Control}

In this section, we review the experimental results for the force-position architecture defined in Section IV. For this experiment, the following force controller parameters were selected for the master controller: $\omega_{f}=50 \mathrm{rad} / \mathrm{s}, \xi_{\mathrm{f}}=1.0$, $\mathrm{f}=0.5 \mathrm{~N}, \beta=1.7 \mathrm{~N}$, and $\tau=40 \mathrm{~ms}$. The slave controller utilized the following position control parameters: $\omega_{p}=50 \mathrm{rad} / \mathrm{s}, \xi_{\mathrm{p}}=0.5, \mathrm{p}=1 \mathrm{~mm}, \beta=3.4 \mathrm{~mm}$, and $\tau=40 \mathrm{~ms}$. The force-position scheme was used with both 3-mode and 7-mode sliding control. The results are charted in Figure V-B. From these results we can see that there is a $44 \%$ improvement in position tracking error and a $20 \%$ improvement in force tracking error for the 7-mode control when compared to the 3-mode control under force-position based control. We can also see from these results that, in terms of the switching activity of the solenoid valves, there is a $27 \%$ reduction in the 7 -mode based control compared to the 3-mode based control.

\section{CONCLUding REMARKS}

We described a pneumatic open-loop actuator system with seven modes of operation based on the state of the on/off solenoid valves. For such a pneumatic system, sliding-mode control laws were developed for position and force control.

The sliding control laws were utilized in the force-position teleoperation architecture. The sliding controllers for the pneumatic system selects one of these seven modes of operation at any given time based on the magnitudes and signs of the switching functions $S_{p}$ and $S_{f}$ for the position and force controllers, respectively.

These closed-loop controls were experimentally verified on a setup consisting of a pair of symmetric pneumatic actuators. For comparison, the experiments were conducted for both the new 7-mode control and the traditional 3- 
mode control It was demonstrated that for this telecoperation architectures, there was a $44 \%$ improvement in position tracking error and up to $27 \%$ reduction in the switching activity with the 7-mode controller when compared to the 3-mode controller. It was also demonstrated that there was a $20 \%$ improvement in force tracking error for the 7 -mode controller when compared to the 3-mode controller.

Having studied the positive effects of 7-mode control (compared to 3-mode control) on positioning accuracy and switching activity in a teleoperation system, the next step would be to examine the effect of time delay on this system.

The air hoses in a pneumatic system can introduce time delay. In this paper, we had neglected the time delays. In general, time delay may destabilize an otherwise stable system. As has been demonstrated in [14], a regular slidingmode controller with some modification can control a slave system to perform a task well independently of time delay. Therefore, future research can explore using sliding-mode control to handle time delays in a pneumatic system.

\section{APPENDIX}

In this appendix, we derive the open-loop model of a pneumatic actuator including its on/off solenoid valves as shown in Figure 1. To describe the air flow dynamics in a cylinder, we assume that:

- Air is an ideal gas and its kinetic energy is negligible in the chambers,

- The pressure and the temperature are homogeneous in each chamber,

- The evolution of the gas in each chamber is polytropic,

- The mass flow rate leakages are negligible, and

- The supply and exhaust pressures are constant.

In this paper, we consider a pneumatic actuator comprised of two chambers as shown in Figure 1. Each chamber is connected to two solenoid valves.

The pressure dynamics of the two chambers of the actuator can be approximated [15] as

$$
\begin{array}{r}
\dot{P_{P}}=\frac{k}{V_{P}}\left(r T_{P} Q_{P}-A_{P} P_{P} \dot{y}\right) \\
\dot{P_{N}}=\frac{k}{V_{N}}\left(r T_{N} Q_{N}+A_{N} P_{N} \dot{y}\right)
\end{array}
$$

where $Q_{P}$ and $Q_{N}$ refer to mass flow rates $(\mathrm{kg} / \mathrm{s})$ of the chambers $P$ and $N$, respectively; $T_{N}$ and $T_{P}$ refer to the temperature $(\mathrm{K})$ in chambers $\mathrm{N}$ and $\mathrm{P}$, respectively. In addition, $V_{P}$ and $V_{N}$ refer to volumes $\left(\mathrm{m}^{3}\right)$ of the chambers and $\mathrm{N}$, respectively, as shown below:

$$
V_{\mathrm{P}}=A_{\mathrm{P}}(1 / 2+\mathrm{y}) \quad \mathrm{V}_{\mathrm{N}}=\mathrm{A}_{\mathrm{N}}(1 / 2-\mathrm{y})
$$

The mass flow rates $Q_{P}$ and $Q_{N}$ can be derived in terms of the discrete voltage inputs $\mathrm{U}_{1}, \mathrm{U}_{2}, \mathrm{U}_{3}$, and $\mathrm{U}_{4}$ shown in Figure 1 and the continuous pressure inputs $\mathrm{P}_{\mathrm{P}}$ and $\mathrm{P}_{\mathrm{N}}$.

$$
Q_{P}=U_{1} Q\left(P_{S}, P_{P}\right)-U_{2} Q\left(P_{P}, P_{E}\right)
$$

As shown below, the model for the mass flow rate has two parameters: the critical pressure ratio $b_{c r i t}$ and the mass flow rate constant $\mathrm{C}_{\mathrm{val}}=\mathrm{C} \rho_{0}$ where $\mathrm{C}$ is the sonic conductance $\left(\mathrm{m}^{3} /(\mathrm{s} \mathrm{Pa})\right)$ and $\rho_{0}$ is the density of air $\left(\mathrm{kg} / \mathrm{m}^{3}\right)$ at a reference condition $\mathrm{T}_{0}=293 \mathrm{~K}[16]$ :

$$
\begin{aligned}
& Q\left(P_{u p}, P_{\text {Down }}\right)=C_{\text {val }} P_{u p} \frac{\overline{T_{\text {Atm }}} \times}{T_{U p}} \times \\
& \overline{1-\frac{\frac{P_{D o w n}}{P U p}-b_{\text {crit }}}{1-b_{\text {crit }}}} \\
& 1 \\
& \text {, if } \frac{P_{\text {Down }}}{P_{\text {U p }}}>b_{\text {crit }} \text { (subsonic) } \\
& \text {, if } \frac{P_{D_{\text {own }}}}{P_{\text {U p }}} \leq b_{\text {crit }} \text { (choked) }
\end{aligned}
$$

The value for $b_{c r i t}=0.433$ comes directly from the data sheet of our solenoid valves, provided by Matrix-Bibus (BIBUS France S.A.S, Chaponnay, France). In the above, $T_{U p}$ is the upstream temperature of air and $T_{A t m}$ is the atmospheric temperature.

It is possible to combine the dynamical equations to write the dynamics of the open-loop pneumatic actuator in a discrete-input form. Differentiating (1) and substituting (A1) and (A2) in it and assuming that the temperature variation in chambers is negligible with respect to the supply temperature (thus $T_{P}=T_{N}=T$ ), the dynamics of the actuator are obtained as the third-order equation

$$
\begin{gathered}
\dddot{y}=f+\frac{k r T}{M} \frac{Q_{P}}{1 / 2+y}-\frac{Q_{N}}{1 / 2-y}+\frac{\dot{\tau}_{E x t}}{M} \\
f=\frac{-b}{M} \ddot{y}-\frac{k}{M} \frac{A_{P} P_{P}}{1 / 2+y}+\frac{A_{N} P_{N}}{1 / 2-y} \dot{y}
\end{gathered}
$$

For each of the discrete modes, the dynamic equation can be obtained by substituting the corresponding mass flow rate terms from (A3a) and (A3b) into (A5). We obtain

$$
\ddot{y}=\begin{array}{ll}
f+\frac{i_{E \times t}}{M} & , \text { mode } M_{1} \\
f+(-1)^{j} g & +\frac{i_{E \times t}}{M}
\end{array}, \text { mode } M_{j}=M_{1}
$$

where the integer $\mathrm{j}$ ranges from 2 to 7 and

$$
\begin{aligned}
g_{2}=\frac{k r T}{M} \frac{Q\left(P_{S}, P_{P}\right)}{(I / 2+y)} & g_{3}=\frac{k r T}{M} \frac{Q\left(P_{P}, P_{E}\right)}{(I / 2+y)} \\
g_{4}=\frac{k r T}{M} \frac{Q\left(P_{N}, P_{E}\right)}{(I / 2-y)} & g_{5}=\frac{k r T}{M} \frac{Q\left(P_{S}, P_{N}\right)}{(I / 2-y)} \\
g_{6}=g_{2}+g_{4} & g_{7}=g_{5}+g_{3}
\end{aligned}
$$

Note that because $P_{E} \leq P_{P} \leq P_{S}, P_{E} \leq P_{N} \leq P_{S}$, $-\mathrm{l} / 2 \leq \mathrm{y} \leq \mathrm{I} / 2$, and mass flow rates are non-negative, the functions $g_{2}$ through $g_{7}$ are all positive or equal to zero.

\section{REFERENCES}

[1] M. Tavakoli, A. Aziminejad, R. Patel, and M. Moallem, "High-fidelity bilateral teleoperation systems and the effect of multimodal haptics," 
IEEE Transactions on Systems, Man and Cybernetics - Part B, vol. 37, no. 6, pp. 1512-1528, December 2007.

[2] J. M. Sabater, M. Hernandez, R. J. Saltarén, R. Aracil, E. Yime, and J. M. Azorín, "Teleoperated parallel climbing robots in nuclear installations," Industrial Robot: An International Journal, vol. 33, no. 5, pp. 381-386, September 2006.

[3] S. Ross, R. Waliany, and M. Swanson, "Inverse kinematics and balance control for intuitive teleoperation of a six-legged mining robot," Robotics Institute, Carnegie Mellon University, Tech. Rep.

[4] M. Q. Le, M. T. Pham, M. Tavakoli, and R. Moreau, "Sliding mode control of a pneumatic haptic teleoperation system with on/off solenoid valves," in Proceedings of 2011 IEEE International Conference on Robotics and Automation (ICRA), Shanghai, China, May 2011.

[5] J. A. Rosas-Flores, J. A. Flores-Campos, and L. G. Corona-Ramírez, "Optimal linearization of the dynamic behavior of an on/off actuated single pneumatic cylinder," in Proceedings of 2008 5th International Conference on Electrical Engineering, Computing Science and Automatic Control (CCE 2008), Mexico City, November 2008, pp. 380385.

[6] A. Girin, F. Plestan, X. Brun, and A. Glumineau, "High-order slidingmode controllers of an electropneumatic actuator: Application to an aeronautic benchmark," International Journal of Control, vol. 79, no. 2, pp. 119-131, 2006.

[7] H. K. Lee, G. S. Choi, and G. H. Choi, "A study on tracking position control of pneumatic actuators," Mechatronics, vol. 12, no. 6, pp. 813$831,2002$.

[8] A. Messina, N. I. Giannoccaro, and A. Gentile, "Experimenting and modelling the dynamics of pneumatic actuators controlled by the pulse width modulation (PWM) technique," Mechatronics, vol. 15, no. 7, pp. 859-881, 2005.

[9] X. Shen, J. Zhang, E. J. Barth, and M. Goldfarb, "Nonlinear modelbased control of pulse width modulated pneumatic servo systems," Journal of Dynamic Systems, Measurement, and Control, vol. 128, pp. 663-669, 2006.

[10] V. I. Utkin, "Sliding mode control design principles and applications to electric drives," IEEE Transactions on Industrial Electronics, vol. 40, no. 1, pp. 23-36, 1993.

[11] T. Nguyen, J. Leavitt, F. Jabbari, and J. E. Bobrow, "Accurate slidingmode control of pneumatic systems using low-cost solenoid valves," IEEE/ASME Transactions on Mechatronics, vol. 12, no. 2, pp. 216219, 2007.

[12] S. Hodgson, M. Q. Le, M. Tavakoli, and M. T. Pham, "Sliding-mode control of nonlinear discrete-input pneumatic actuators," in Proceedings of IEEE/RSJ International Conference on Intelligent Robots and Systems (IROS), San Francisco, USA, September 2011, pp. 738-743.

[13] M. Q. Le, M. T. Pham, M. Tavakoli, and R. Moreau, "An enhanced sliding-mode control for a pneumatic-actuated teleoperation system," in Proceedings of IEEE/RSJ International Conference on Intelligent Robots and Systems (IROS), San Francisco, USA, September 2011, pp. 659-664.

[14] J. H. Park and H. C. Cho, "Sliding-mode controller for bilateral teleoperation with varying time delay," in Proceedings of IEEE/ASME International Conference on Advanced Intelligent Mechatronics, Atlanta, USA, September 1999, pp. 311-316.

[15] M. Q. Le, M. T. Pham, R. Moreau, and T. Redarce, "Transparency of a pneumatic teleoperation system using on/off solenoid valves," in Proceedings of 2010 19th IEEE International Symposium on Robot and Human Interactive Communication, Viareggio, Italy, September 2010, pp. 15-20.

[16] P. Beater, Pneumatic Drives - System Design, Modelling and Control. Berlin: Springer, 2006. 